\title{
Transport properties of low-dimensional semiconductor structures in the presence of spin-orbit interaction
}

\author{
P.M. Krstajić*, M. Pagano, P. Vasilopoulos \\ Department of Physics, Concordia University, 7141 Sherbrooke Ouest, Montréal, Québec, Canada H4B 1R6
}

\section{A R T I C L E I N F O}

\section{Article history:}

Received 1 September 2010

Received in revised form

1 November 2010

Accepted 5 November 2010

\begin{abstract}
A B S T R A C T
Transport properties of a two-dimensional electron gas (2DEG) and of quantum wires are theoretically studied in the presence of both Rashba and Dresselhaus terms of the spin-orbit interaction (SOI). Fully quantum mechanical expressions for the conductivity are evaluated for very low temperatures and the differences between them and previous semiclassical results are highlighted. Two kinds of confining potentials in quantum wires are considered, square-type and parabolic. Various cases depending on the relative strengths of two different SOI terms are discussed and the relaxation times for various impurity potentials are evaluated. In addition, the spin accumulation in a 2DEG and in a quantum wire $(\mathrm{QW})$ is evaluated semiclassically and its dependence on the Fermi energy and the SOI strengths is discussed. A nearly saw-tooth dependence on the electron concentration is obtained for a $\mathrm{QW}$ with parabolic confinement.
\end{abstract}

(C) 2010 Published by Elsevier B.V.

\section{Introduction}

The investigation of spin-dependent phenomena has intensified since the discovery of giant magnetoresistance in 1988 [1] due to their potential applications in novel devices [2]. Particular attention has been given to the spin-orbit effects in semiconductor structures. It is known that the spin-orbit interaction (SOI) may play an important role in the transport properties of low-dimensional semiconductor structures. The SOI may manifest itself in semiconductor structures either as a result of the breaking of macroscopic inversion symmetry of the whole structure, referred to as the Rashba SOI (RSOI) term [3], or due to the lack of inversion symmetry of the crystal structure, referred to as the Dresselhaus SOI (DSOI) term $[4,5]$. The RSOI term depends on band alignment and on any applied external potential if it breaks the overall inversion symmetry which means it can be tuned by applying a bias [6-8]. On the other hand, the DSOI term is present in bulk materials and semiconductor heterostructures. Some III-V semiconductors, such as GaSb, exhibit large spin-splitting in the absence of a magnetic field, due to the difference between cations and anions.

The transport properties of low-dimensional semiconductor structures made of materials with pronounced spin-orbit effects are expected to be different than those of structures lacking or negligible zero field spin-splitting. They have been studied in the past either in the presence of a magnetic field [9] or by numerical methods and mostly when the RSOI is present [10]. In addition, weak-localization corrections to the conductivity of a 2DEG have been studied in Ref. [11] with the RSOI and DSOI terms taken into

Q2 * Corresponding author

Q1 E-mail address: predrag222@gmail.com (P.M. Krstajić). account. In this work we consider both the RSOI and DSOI terms and derive approximate analytical expressions for the diffusive conductivity of a 2DEG at very low temperatures and for a quasi-onedimensional electron gas (1DEG), i.e., for a quantum wire (QW), with square or parabolic confinement. The non-diagonal contribution to the conductivity is evaluated numerically.

The case of equal RSOI and DSOI strengths is treated in some detail since it was predicted [12] that the role of spin-independent scattering is reduced. This is actually correct when the cubic DSOI term is neglected as confirmed by a recent experiment [13]. We underline the differences between previous semiclassical results and our fully quantum mechanical ones. Further, we provide explicit expressions for the relaxation time, due to impurity scattering, that most frequently has been taken as a constant in the literature.

We organize the paper as follows. In Section 2 we present the theoretical model and give the relevant expressions of the eigenvalues and eigenvectors for a 2DEG and a 1DEG. We also give the general one-particle expressions for the dc conductivities. We present and discuss the results in Section 3 and summarize our conclusions in Section 4.

\section{Theoretical model}

\subsection{A $2 D E G$}

We first consider a 2DEG, in the $(x, y)$ plane, in the presence of both Rashba and Dresselhaus terms of the SOI with strengths, respectively, $\alpha$ and $\beta$. The relevant one-electron Hamiltonian is

$H=H_{0}+H_{R}+H_{D}$ doi:10.1016/j.physe.2010.11.008 
where $H_{0}=\hbar^{2} k^{2} / 2 m^{*}$ is the free-electron term and $m^{*}$ the effective mass. $H_{R}$ and $H_{D}$ are, respectively, the Rashba and Dresselhaus SOI terms given by

$H_{R}=\alpha\left(\sigma_{x} k_{y}-\sigma_{y} k_{x}\right), \quad H_{D}=\beta\left(\sigma_{x} k_{x}-\sigma_{y} k_{y}\right)$.

The term $H_{D}$ given above is for a 2DEG grown along the [l 001 l direction. If grown along different directions it acquires a different form, see, e.g., Ref. [14] for growth along the [ $\left[\begin{array}{lll}0 & 1 & 0\end{array}\right]$, [ $\left.\begin{array}{llll}1 & 1 & 0\end{array}\right]$, and [ 1 - 10$]$ directions.

The eigenvalues of Eq. (1) are [15]

$E_{k}^{\sigma}=E_{k}^{ \pm}=\frac{\hbar^{2} k^{2}}{2 m^{*}} \pm\left[\left(\alpha k_{y}+\beta k_{x}\right)^{2}+\left(\alpha k_{x}+\beta k_{y}\right)^{2}\right]^{1 / 2}$,

and the eigenvectors

$|\mathbf{k}, \sigma\rangle=\frac{e^{i\left(k_{x} x+k_{y} y\right)}}{\sqrt{2}}\left(\begin{array}{c}1 \\ \sigma e^{i \phi}\end{array}\right), \quad \tan \phi=\frac{-\left(\alpha k_{x}+\beta k_{y}\right)}{\alpha k_{y}+\beta k_{x}}$,

with $\sigma= \pm$ being the spin index. It is convenient to express the eigenvalues in polar coordinates $k$ and $\theta$. Then Eq. (3) is rewritten as

$E_{k}^{ \pm}=\lambda k^{2} \pm k \varepsilon_{\theta}, \quad \varepsilon_{\theta}=\left[\alpha^{2}+\beta^{2}+2 \alpha \beta \sin (2 \theta)\right]^{1 / 2}$,

where $\lambda=\hbar^{2} / 2 m^{*}$ and $\tan \theta=k_{y} / k_{x}$. At fixed energy, usually the Fermi energy $E_{F}$, there are two characteristic wave vectors $k_{ \pm}$

$k_{ \pm}=\left[\mp \varepsilon_{\theta}+\sqrt{\varepsilon_{\theta}^{2}+4 \lambda E_{F}}\right] / 2 \lambda$.

In what follows we will need the matrix elements of the velocity operator, along $x$ and $y$ axis,

$v_{x}=\frac{1}{\hbar} \frac{\partial H}{\partial k_{x}}=\frac{1}{\hbar}\left(\begin{array}{cc}2 \lambda k_{x} & \beta+i \alpha \\ \beta-i \alpha & 2 \lambda k_{x}\end{array}\right)$,

and

$v_{y}=\frac{1}{\hbar} \frac{\partial H}{\partial k_{y}}=\frac{1}{\hbar}\left(\begin{array}{cc}2 \lambda k_{y} & \alpha+i \beta \\ \alpha-i \beta & 2 \lambda k_{y}\end{array}\right)$

For the evaluation of the conductivity we need the matrix elements of the velocity operator $\hat{v}_{\mu}, \mu=x, y$.

The diagonal elements in the spin index are

$\left\langle\mathbf{k}, \sigma\left|\hat{v}_{x}\right| \mathbf{k}, \sigma\right\rangle=\left[2 \lambda k_{x} \pm\left[\left(\alpha^{2}+\beta^{2}\right) k_{x}+2 \alpha \beta k_{y}\right] / k \varepsilon_{\theta}\right] / \hbar$,

and the off-diagonal ones

$\left\langle\mathbf{k},+\left|\hat{v}_{x}\right| \mathbf{k},-\right\rangle=\left\langle\mathbf{k},-\left|\hat{v}_{x}\right| \mathbf{k},+\right\rangle^{*}=\left[i\left(\alpha^{2}-\beta^{2}\right) k_{y} / k \varepsilon_{\theta}\right] / \hbar$.

Similar expressions can be derived for the matrix elements of the operator $\hat{v}_{y}$.

\subsection{A quasi-1DEG}

We now consider a quasi-1DEG, such as a QW, with confinement along the $x$ axis.

Square confinement. We assume that the confining potential is zero inside the QW and infinitely high at its walls. If only the first subband is occupied, the energy spectrum is given by [16]

$E_{k_{y}}^{\sigma}=E_{k_{y}}^{ \pm}=E_{1}+\lambda k_{y}^{2} \pm\left(\alpha^{2}+\beta^{2}\right)^{1 / 2} k_{y}$

and the wave function acquires the simple form

$\psi_{\sigma}(x, y)=\frac{1}{\sqrt{2}}\left(\begin{array}{c}1 \\ \sigma e^{i \phi}\end{array}\right), \quad \tan \phi=-\beta / \alpha$.

Only the operator $\hat{v}_{y}$ is relevant and its diagonal matrix elements are

$\left\langle k_{y}, \sigma\left|\hat{v}_{y}\right| k_{y}, \sigma\right\rangle=\left[2 \lambda k_{y} \pm\left(\alpha^{2}+\beta^{2}\right)^{1 / 2}\right] / \hbar ;$

its off-diagonal elements are equal to zero.
Parabolic confinement, $\alpha=\beta$. The transport properties of $\mathrm{QW}$ with parabolic confinement have already been treated in the literature [17] but with different SOI term, or taking into account only the Rashba term [18]. If $V(x)$ is the confining potential, Eq. (1) is written as

$H=H_{0}+V(x)+H_{R}+H_{D}$

With $V(x)=m^{*} \omega^{2} x^{2} / 2$ analytical solutions exist [12] for $\alpha= \pm \beta$ and the eigenfunctions of Eq. (14) for $\alpha=\beta$ have the form

$\Psi_{\sigma}(x, y)=\frac{1}{\sqrt{2}}\left(\begin{array}{c}1 \\ \sigma e^{-i \pi / 4}\end{array}\right) \phi(x, y) e^{-\sigma\left(i \sqrt{2} \alpha m / \hbar^{2}\right)(x+y)}$,

while $\phi(x, y)$ satisfies the spin-independent equation

$\left[-\lambda \nabla^{2}+V(x, y)\right] \phi(x, y)=\left(E+\alpha^{2} / \lambda\right) \phi(x, y)$.

With $V(x, y) \equiv V(x)=m^{*} \omega^{2} x^{2} / 2$ the solution is

$\phi(x, y)=N_{n} H_{n}(x / l) e^{-x^{2} / 2 l^{2}} e^{i k_{y} y}$,

where $N_{n}=\left[2^{n} n ! \sqrt{\pi} l\right]^{-1 / 2}$ and $l=\left[\hbar / m^{*} \omega\right]^{1 / 2}$. Then, with $E_{y}=$ $\hbar^{2} k_{y}^{2} / 2 m^{*}$, the eigenvalues are

$E_{n}=(n+1 / 2) \hbar \omega+E_{y}-2 \alpha^{2} m^{*} / \hbar^{2}$.

Only the diagonal matrix elements of $\hat{v}_{y}$ are relevant here since its off-diagonal elements vanish. They are

$\left\langle k_{y}, \sigma\left|\hat{v}_{y}\right| k_{y}, \sigma\right\rangle=2 \lambda k_{y} / \hbar$.

Parabolic confinement, $\alpha=-\beta$. When the two SOI interaction strengths have opposite sign but the same magnitude, the wave function have somewhat different form than Eq. (20)

$\Psi_{\sigma}(x, y)=\frac{1}{\sqrt{2}}\left(\begin{array}{c}1 \\ \sigma e^{5 i \pi / 4}\end{array}\right) \phi(x, y) e^{-\sigma\left(i \sqrt{2} \alpha m / \hbar^{2}\right)(x-y)}$.

However, the energy and the matrix elements of $\hat{v}_{y}$ have the same form and so does the diagonal conductivity. The off-diagonal matrix elements are again zero. For this reason we will not go further into consideration of this case.

Parabolic confinement, weak $\alpha$ and $\beta$. The analytical solution for the eigenvalues and eigenvectors are not known for unequal Rashba and Dresselhaus SOI strengths $\alpha \neq \beta$, so one may resort to approximate or numerical methods. We treat [19] $H_{S O}$ as a perturbation and expand the wave function in terms of the (unperturbed) eigenfunctions $\phi_{n}(x)$ of $H_{0}$, i.e.,

$\Psi(x, y)=\sum_{n, \sigma} A_{n}^{\sigma} \phi_{n}(x) \chi_{\sigma} e^{i k_{y} y}$,

where $\chi_{\sigma}$ are the eigenvectors of $\sigma_{z}$, that is $(1,0)^{T}$ and $(0,1)^{T}$. The eigenfunctions of $H_{0}$ are harmonic oscillator functions. The matrix elements of the interaction term are non-zero only between states of opposite spin. The diagonal matrix elements are

$\left(H_{S O}\right)_{n n}^{ \pm, \mp}=(\alpha \pm i \beta) k_{y}$,

and the off-diagonal ones

$\left(H_{S O}\right)_{n m}^{+,-}=\frac{\alpha-i \beta}{l \sqrt{2}}\left[\sqrt{n+1} \delta_{n, m-1}-\sqrt{n} \delta_{n, m+1}\right]$,

since $k_{x}$ mixes adjacent states, and

$\left(H_{S O}\right)_{n m}^{-,+}=\frac{-\alpha-i \beta}{l \sqrt{2}}\left[\sqrt{n+1} \delta_{n, m-1}-\sqrt{n} \delta_{n, m+1}\right]$. 
We will consider only the first three states. This leads to the secular equation, $\operatorname{det}|S-E I|=0$ where

$$
S=\left(\begin{array}{cccccc}
\varepsilon_{0} & \gamma_{+} k_{y} & 0 & \frac{\gamma_{-}}{\sqrt{2 l}} & 0 & 0 \\
\gamma_{-} k_{y} & \varepsilon_{0} & \frac{-\gamma_{+}}{\sqrt{2 l}} & 0 & 0 & 0 \\
0 & \frac{-\gamma_{-}}{\sqrt{2 l}} & \varepsilon_{1} & \gamma_{+} k_{y} & 0 & \frac{\gamma_{+}}{T} \\
\frac{\gamma_{+}}{\sqrt{2 l}} & 0 & \gamma_{-} k_{y} & \varepsilon_{1} & \frac{-\gamma_{+}}{l} & 0 \\
0 & 0 & 0 & \frac{-\gamma_{-}}{l} & \varepsilon_{2} & \gamma_{+} k_{y} \\
0 & 0 & \frac{\gamma_{+}}{l} & 0 & \gamma_{-} k_{y} & \varepsilon_{2}
\end{array}\right) \text {, }
$$

$\gamma_{ \pm}=\alpha \pm i \beta$, and $\varepsilon_{n}=(n+1 / 2) \hbar \omega+\lambda k_{y}^{2}$. The energy spectrum (the first three levels) is shown in Fig. 1 for two different values of the SOI strengths, (a) $\alpha=\beta=\alpha_{0}$, (b) $\alpha=\alpha_{0}, \beta=0.5 \alpha_{0}$, where $\alpha_{0}=5 \times$ $10^{-11} \mathrm{eV} \mathrm{m}$ (Ref. [8]). Anticrossings are visible in the latter case when the two SOI strengths are different. The existence of two sets of curves is due to the presence of SOI that lifts the spin degeneracy. The energy difference between the levels is taken to be $E_{0}=\hbar \omega=0.5 \mathrm{meV}$

\subsection{Dc conductivities}

Within the one-electron approximation and for small electric fields the conductivity tensor $\sigma_{\mu v}(\mu, v=x, y, z)$ may be separated into diagonal and non-diagonal parts $\sigma_{\mu v}=\sigma_{\mu v}^{d}+\sigma_{\mu v}^{\text {nd }}$. The diffusive contribution $\sigma_{\mu \nu}^{d i f}$ to $\sigma_{\mu \nu}^{d}$ is [20]

$\sigma_{\mu \nu}^{d i f}=\frac{e^{2}}{k_{B} T S_{0}} \sum_{\zeta} f_{\zeta}\left(1-f_{\zeta}\right) \tau\left(E_{\zeta}\right) v_{\mu \zeta} v_{v \zeta}$,

where $\zeta=\left(\sigma, k_{x}, k_{y}\right)$ denotes the set of quantum numbers, $S_{0}$ the area, $k_{B}$ the Boltzmann constant $v_{\mu}^{\zeta}=\left\langle\zeta\left|v_{\mu}\right| \zeta\right\rangle$ the velocity
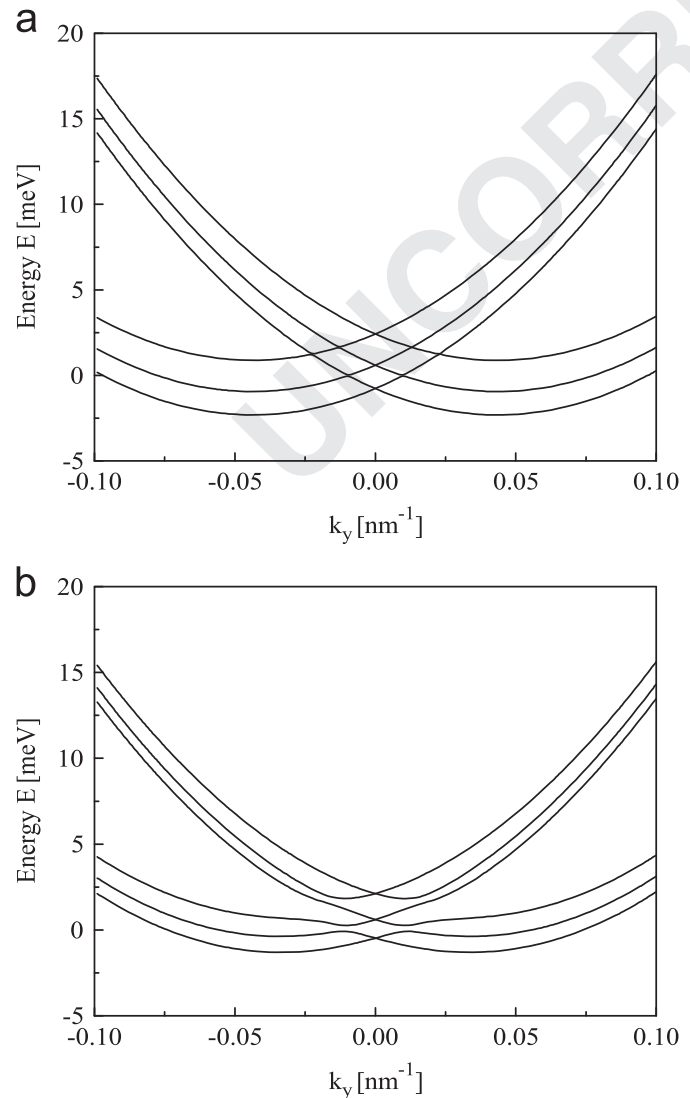

Fig. 1. Energy spectrum for a QW with parabolic confinement for two different values of the SOI strengths, (a) $\alpha=\beta=\alpha_{0}$ and (b) $\alpha=\alpha_{0}, \beta=0.5 \alpha_{0}$, where $\alpha_{0}=5 \times 10^{-11} \mathrm{eV} \mathrm{m}$. expectation value in the state $\zeta$, and $f_{\zeta}$ the Fermi-Dirac distribution. Eq. (26) is valid only for elastic scattering. The collisional contribution to $\sigma_{\mu \nu}^{d}$ is [20]

$\sigma_{\mu \nu}^{c o l}=\frac{e^{2}}{2 S_{0} k_{B} T} \sum_{\zeta \zeta^{\prime}} f_{\zeta}\left(1-f_{\zeta^{\prime}}\right) W_{\zeta \zeta^{\prime}}\left(\alpha_{\mu}^{\zeta}-\alpha_{\mu}^{\zeta^{\prime}}\right)^{2}$,

for both elastic $\left(f_{\zeta}=f_{\zeta^{\prime}}\right)$ and inelastic $\left(f_{\zeta} \neq f_{\zeta^{\prime}}\right)$ scattering. $W_{\zeta \zeta^{\prime}}$ is the transition rate between the unperturbed one-electron states $|\zeta\rangle$ and $\left|\zeta^{\prime}\right\rangle$, and $\alpha_{\mu}^{\zeta}=\left\langle\zeta\left|r_{\mu}\right| \zeta\right\rangle$ the expectation value of the $\mu$-component of the position operator $r$ in the state $|\zeta\rangle$.

The dc non-diagonal contribution $\sigma_{\mu \nu}^{n d}$ to the conductivity is given by [20]

$\sigma_{\mu v}^{n d}=\frac{i \hbar e^{2}}{S_{0}} \sum_{\zeta \neq \zeta^{\prime}} f_{\zeta^{\prime}}\left(1-f_{\zeta^{\prime}}\right) v_{v \zeta^{\prime} \zeta^{\prime}} v_{\mu \zeta^{\prime} \zeta} \times \frac{1-e^{\left(E_{\zeta^{\prime}}-E_{\zeta^{\prime}}\right) / k_{B} T}}{E_{\zeta}-E_{\zeta^{\prime}}} \lim _{\varepsilon \rightarrow 0} \frac{1}{E_{\zeta}-E_{\zeta^{\prime}}+i \varepsilon}$

If we use the identity $f_{\zeta}\left(1-f_{\zeta^{\prime}}\right) \exp \left[\beta\left(E_{\zeta^{\prime}}-E_{\zeta^{\prime}}\right)\right]=f_{\zeta^{\prime}}\left(1-f_{\zeta}\right)$, Eq. (28) takes the form of the well-known Kubo-Greenwood formula

$\sigma_{\mu \nu}^{n d}=\frac{i \hbar e^{2}}{S_{0}} \sum_{\zeta \zeta^{\prime}} \frac{\left(f_{\zeta}-f_{\zeta^{\prime}}\right) v_{v \zeta \zeta^{\prime}} v_{\mu \zeta^{\prime} \zeta}}{\left(E_{\zeta}-E_{\zeta^{\prime}}\right)\left(E_{\zeta}-E_{\zeta^{\prime}}+i \Gamma_{\zeta}\right)}$,

with $\zeta \neq \zeta^{\prime}$ and $\varepsilon$ taken as the level width $\Gamma_{\zeta}$.

\subsection{Spin accumulation}

In order to study the spin accumulation in the structures of interest one should first calculate the net spin in the presence of a weak electric field $E_{e l}$. Then from the semiclassical Boltzmann equation one finds [22]

$\left\langle S_{x, y}\right\rangle=\frac{e \hbar \tau}{m^{*}} \sum_{\sigma} \int \frac{d^{d} k}{(2 \pi)^{d}} \mathbf{E}_{e l} \cdot \mathbf{k} s_{\sigma x, y} \frac{\partial f_{\sigma}}{\partial E}$,

where $d=1,2$ for a QW and a 2DEG, respectively; $s_{\sigma x, y}$ are the diagonal matrix elements of the spin operator. The electric field is assumed to point in an arbitrary direction. At small temperatures the derivative of the distribution function with respect to the energy may be replaced by a $\delta$ function. The result, as explained in Ref. [22], is that Eq. (30) is valid when the Fermi, thermal, and SOI energies satisfy the inequalities $k_{F} \varepsilon_{\theta} \ll k_{B} T \ll E_{F}$.

\section{Results and discussion}

\section{1. $2 D E G$}

We first evaluate the diagonal contributions to the conductivity. The collisional contribution $\sigma_{\mu \nu}^{c o l}$ vanishes identically because $\alpha_{\mu}^{\zeta}$ is independent of the state $\zeta$ and thus $\alpha_{\mu}^{\zeta}-\alpha_{\mu}^{\zeta^{\prime}}=0$. Now it can be proven [23] that $\sigma_{x x}^{\text {dif }}=\sigma_{y y}^{\text {dif }}$ so that one can write $\sigma_{x x}^{\text {dif }}=1 / 2\left(\sigma_{x x}^{\text {dif }}+\sigma_{y y}^{\text {dif }}\right)$ in order to simplify the results. An approximate analytic expression can be found near zero temperature, when $f_{\zeta}\left(1-f_{\zeta}\right) / k_{B} T$ is replaced by the Dirac $\delta$-function in Eq. (26). Further, one may replace $\tau\left(E_{\zeta}\right)$ in Eq. (26) by some constant mean value $\tau_{F}$ at the Fermi level, which is quite reasonable approximation for a Fermi degenerate gas [21]. Thus, the result is

$\sigma_{x x}^{d i f}=\frac{e^{2} \pi \tau_{F}}{h^{2} \lambda}\left[2\left(\alpha^{2}+\beta^{2}+2 \lambda E_{F}\right)-\left|\alpha^{2}-\beta^{2}\right|\right]$,

which holds for $\alpha, \beta>0$. The first term in Eq. (31) is the same as the one obtained from the semiclassical Boltzmann equation in Refs. [22,23] for $\delta$-function scattering. The additional term $\propto\left|\alpha^{2}-\beta^{2}\right|$ represents a quantum mechanical contribution to the conductivity. We will examine two special cases in more detail: (i) equal SOI strengths $(\alpha=\beta)$ and (ii) absence of the Dresselhaus term $(\alpha \neq 0, \beta=0)$.
111 
(i) For $\alpha=\beta$ the energy spectrum consists of two identical paraboloids [24] with $E_{k}^{ \pm}=\lambda k^{2} \pm \sqrt{2} \alpha k[1+\sin (2 \theta)]^{1 / 2}$. Then the result for $\sigma_{x x}^{\text {dif }}$, Eq. (31), acquires the simple form

$\sigma_{x x}^{\text {dif }}=\frac{4 e^{2} \tau_{F} \pi}{h^{2}}\left(E_{F}+\alpha^{2} / \lambda\right)$.

Due to the SOI the bottom of the conduction bands is shifted by an energy $|\Delta E|=\alpha^{2} / \lambda$ and the electron concentration of the perturbed conduction bands is

$n_{0}^{\prime}=\frac{m^{*}}{\pi \hbar^{2}}\left(E_{F}+\alpha^{2} / \lambda\right)$.

The last relation can be used to obtain the Drude form of the conductivity

$\sigma_{x x}^{\text {dif }}=n^{\prime}{ }_{0} e^{2} \tau_{F} / m^{*}$

(ii) If the Dresselhaus SOI term is absent, i.e., for $\beta=0$, the conductivity assumes a simpler form than Eq. (31),

$\sigma_{x x}^{\text {dif }}=\frac{n_{0} e^{2} \tau_{F}}{m^{*}}+\frac{m^{*} e^{2} \tau_{F}}{2 \pi \hbar^{4}} \alpha^{2}$

by virtue of the relation between the concentration $n_{0}$ of an unperturbed 2DEG (in the absence of SOI) and the Fermi energy, $n_{0}=\left(m^{*} / \pi \hbar^{2}\right) E_{F}$. This relation can also be recast in a more familiar, Drude-type form if one takes into account the shift $|\Delta E|=\alpha^{2} / 4 \lambda$ of the conduction band minima,

$\sigma_{x x}^{\text {dif }}=n^{\prime}{ }_{0} e^{2} \tau_{F} / m^{*}$

We emphasize that both branches are included and shifted downwards by the same amount. Before we proceed with the evaluation of the conductivity, it would be appropriate to evaluate the transport scattering time for elastic scattering. First, we consider the case of equal Rashba and Dresselhaus SOI terms, i.e., $\alpha=\beta$. Then, using the definition Eq. (A.1) in Appendix A we obtain, similar to Eq. (A.4), the result

$\frac{1}{\tau_{F}}=\frac{\pi n_{i} U_{0}^{2}}{\hbar \lambda}\left(A_{\theta}^{+}+A_{\theta}^{-}\right)$

with $A_{\theta}^{ \pm}$being the following integrals over $\theta$ :

$A_{\theta}^{ \pm}=\int_{0}^{2 \pi} d \theta\left(1 \mp \frac{\alpha \sqrt{B_{\theta}}}{\sqrt{\alpha^{2} B_{\theta}+C_{ \pm}}}\right) \frac{1-\cos \theta}{k_{s}^{2}+k_{ \pm}^{2}+k_{0}^{2}-2 k_{ \pm} k_{0} \cos \theta} ;$

here $B_{\theta}=2+2 \sin 2 \theta, C_{ \pm}=4 \lambda k_{ \pm}\left(\lambda k_{ \pm} \pm \sqrt{2} \alpha\right), k_{ \pm}$are the wave vectors corresponding to the Fermi energy, given by Eq. (6), and $U_{0}$ is the Fourier transform of the scattering potential. The integration in Eq. (38) is carried out numerically and the value of $k_{0}$ is determined by the root of $E_{k}^{\sigma}=E_{k^{\prime}}^{\sigma}$, with $\hbar k^{\prime}$ being the momentum after scattering. Analytical expressions are available when the Rashba SOI dominates over the Dresselhaus SOI term, i.e., for $\alpha \gg \beta$, see Eqs. (A.4)-(A.6) in Appendix A. For convenience we rewrite here the result for impurities having a screened Coulomb potential

$\frac{1}{\tau_{F}}=\frac{2 \pi^{2} n_{i} U_{0}^{2}}{\hbar}\left(I_{C+}+I_{C-}\right)$

where $I_{C+}$ and $I_{C_{-}}$are specified in Appendix $\mathrm{A}$ and $n_{i}$ is the concentration of impurities per unit area. This result is derived under the assumption $2 \lambda k_{-}>\alpha$. For typical values $n_{i} \approx 10^{9} \mathrm{~cm}^{-2}$, $n_{2 \mathrm{D}} \approx 10^{11} \mathrm{~cm}^{-2}$, and $k_{\mathrm{s}} \approx 10^{8} \mathrm{~m}^{-1}$ the scattering time is about $\tau \approx 3$ ps. In Fig. 2 we show the dependence of the longitudinal conductivity $\sigma_{x x}^{d}$ of the 2DEG as a function of its concentration for equal Rashba and Dresselhaus SOI terms (solid curve) and in the presence of only the Rashba SOI term (dashed curve). The conductivity increases with the concentration of the 2DEG in both cases. The difference in values of $\sigma_{x x}^{d}$ in the two cases is not

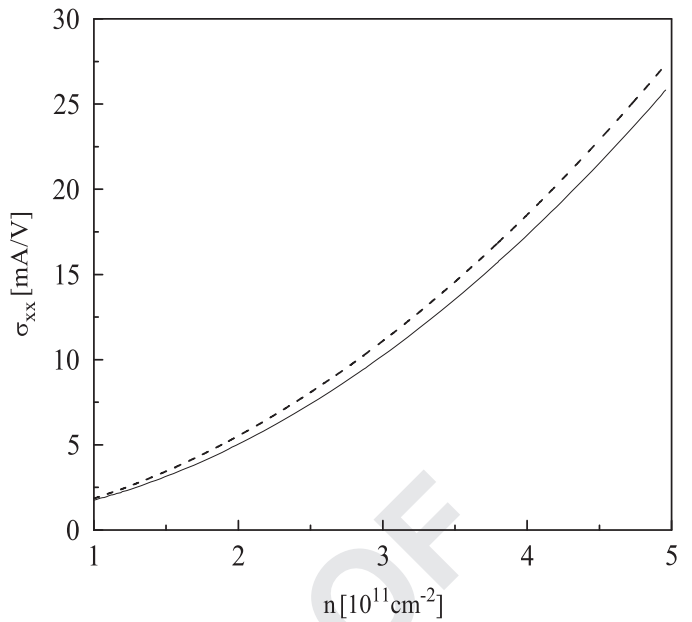

Fig. 2. Conductivity $\sigma_{x x}^{d}$ of the $2 \mathrm{DEG}$ as a function of electron concentration when the Dresselhaus and Rashba SOI term are equal, $\alpha=\beta=\alpha_{0}$ (solid curve) and in the absence of Dresselhaus SOI term, $\beta=0, \alpha=\alpha_{0}$ (dashed curve), where $\alpha_{0}=5 \times 10^{-11} \mathrm{eV} \mathrm{m}$.

Fig. 3. Conductivity of a 2DEG as a function of the strength $\alpha$, at zero temperature, for equal Rashba and Dresselhaus SOI terms, $\alpha=\beta$ (solid curve), and for $\alpha \neq 0$, and $\beta=0$ (dashed curve). The electron concentration was kept constant, $n_{0}=1 \times 10^{11} \mathrm{~cm}^{-2}$.

significant since the Fermi energy depends on the SOI too which compensates in part the absence of the Dresselhaus SOI term. The value of $\alpha(\beta)$ is taken to be $\alpha_{0}=5 \times 10^{-11} \mathrm{eV} \mathrm{m}$.

Further, it would be useful to investigate the dependence of the conductivity on the strength $\alpha$. Fig. 3 shows this dependence for equal Rashba and Dresselhaus SOI terms (solid curve), for the Rashba SOI term only (dashed curve), and for $\alpha=0.5 \beta$ (dotted curve) versus $\alpha$ at zero temperature and fixed electron concentration, $n_{2 \mathrm{D}}=10^{11} \mathrm{~cm}^{-2}$. The functional dependence is not linear since both the Fermi energy $E_{F}$ and the scattering time $\tau$ depend on $\alpha$. In fact, the increase of the conductivity when only the Rashba term is present is mainly due to the increase in the relaxation time.

Next, we proceed with the non-diagonal part of the conductivity $\sigma^{\text {nd }}$ given by Eq. (29). The summation in Eq. (29) is over different spin indices $\sigma \neq \sigma^{\prime}$. The parameter $\Gamma_{\sigma \sigma^{\prime}}$, characterizing the energy level broadening, is assumed to be independent of $\operatorname{spin} \Gamma_{\sigma \sigma^{\prime}} \equiv \Gamma$. The fraction in Eq. (29) can be expanded and Eq. (29) can be rewritten as

$\sigma_{x x}^{n d}=\frac{i \hbar e^{2}}{S_{0}} \sum_{\mathbf{k} \sigma \sigma^{\prime}} \frac{f_{\mathbf{k} \sigma}-f_{\mathbf{k} \sigma^{\prime}}}{E_{\mathbf{k}}^{\sigma}-E_{\mathbf{k}}^{\sigma^{\prime}}} \frac{\left(E_{\mathbf{k}}^{\sigma}-E_{\mathbf{k}}^{\sigma^{\prime}}-i \Gamma\right) v_{x \mathbf{k} \sigma \sigma^{\prime}} v_{x \mathbf{k} \sigma^{\prime} \sigma}}{\left(E_{\mathbf{k}}^{\sigma}-E_{\mathbf{k}}^{\sigma^{\prime}}\right)^{2}+\Gamma^{2}}$. 
1 It can be shown that the real part of the sum in Eq. (40) vanishes, so only its imaginary part, $\propto i \Gamma$, survives. Furthermore, the product of the velocity matrix elements is (see Eq. (10))

$v_{x \mathbf{k}+-} v_{x \mathbf{k}-+}=v_{x \mathbf{k}-+} v_{x \mathbf{k}+-}=\frac{\left(\alpha^{2}-\beta^{2}\right)^{2} \sin ^{2} \theta}{\hbar^{2} \varepsilon_{\theta}^{2}}$.

Taking into account the fact that the first fraction under the sum in Eq. (40) does not change sign upon interchanging the spin indices $\sigma$ and $\sigma^{\prime}$, Eq. (40) becomes

$\sigma_{x x}^{n d}=\frac{e^{2}}{2 h} \frac{\left(\alpha^{2}-\beta^{2}\right)^{2}}{\pi} \int d k d \theta \frac{f_{\mathbf{k}+}-f_{\mathbf{k}-}}{4 k^{2} \varepsilon_{\theta}^{2}+\Gamma^{2}} \frac{\Gamma \sin ^{2} \theta}{\varepsilon_{\theta}^{3}}$.

The level broadening $\Gamma$ is estimated by the golden rule $\Gamma \approx \hbar \sum_{\zeta \zeta^{\prime}} W_{\zeta \zeta^{\prime}}$. At zero temperature the integral over the momentum $k$ can be carried out analytically. The result is

$$
\begin{aligned}
\sigma_{x x}^{n d}= & \frac{e^{2}}{4 h} \frac{\left(\alpha^{2}-\beta^{2}\right)^{2}}{\pi} \int_{0}^{2 \pi} d \theta \frac{\sin ^{2} \theta}{\varepsilon_{\theta}^{4}} \\
& \times\left[\arctan \left(2 \varepsilon_{\theta} k^{-} / \Gamma\right)-\arctan \left(2 \varepsilon_{\theta} k^{+} / \Gamma\right)\right],
\end{aligned}
$$

where

$k^{ \pm}=\left[\mp \varepsilon_{\theta}+\left(\varepsilon_{\theta}^{2}+4 \lambda E_{F}\right)^{1 / 2}\right] / 2 \lambda$.

In Fig. 4 we show the numerically evaluated conductivity $\sigma_{x x}^{\text {nd }}$ as a function of the level broadening $\Gamma$. The solid black curve is the result from Eq. (42), for $T=10 \mathrm{~K}$, and the dashed one for $T=0 \mathrm{~K}$ using Eq. (43), for the interaction strengths $\alpha=5 \times 10^{-11} \mathrm{eV} \mathrm{m}$, $\beta=0.5 \alpha$.

We proceed with the evaluation of the spin accumulation in a 2DEG in the presence of SOI. If we denote the angle between the electric field $\mathbf{E}_{\mathbf{e l}}$ and the wave vector $\mathbf{k}$ with $\psi$ and use Eq. (30), it is possible to find an analytical expression for the net spin components. Along the $x$ axis the result is

and along the $y$ axis

$\left\langle S_{y}\right\rangle=\frac{e E_{e l} m^{*} \tau_{F}}{2 \pi \hbar^{3}}(\alpha \cos \psi+\beta \sin \psi)$

43

45

Fig. 4. Non-diagonal conductivity of a 2DEG, with the RSOI and DSOI terms present, as a function of the level broadening $\Gamma$. The solid black curve is the exact numerical result from Eq. (42), for $T=10 \mathrm{~K}$, and the dashed one for $T=0 \mathrm{~K}$ using Eq. (43). The $\left\langle S_{x}\right\rangle=\frac{e E_{e l} m^{*} \tau_{F}}{2 \pi \hbar^{3}}(\beta \cos \psi+\alpha \sin \psi)$,

\subsection{Quasi-1D system}

$\sigma_{y y}^{d i f}=\frac{4 e^{2} \tau_{F}}{\pi \hbar^{2}} \sum_{n}\left[\lambda\left(E_{F}-E_{n}^{0}\right)+\alpha^{2}\right]^{1 / 2}$, interaction strengths are $\alpha=5 \times 10^{-11} \mathrm{eV} \mathrm{m}, \beta=0.5 \alpha$.
The magnitude of the spin accumulation $\langle S\rangle$ is then $\langle S\rangle=$ $\left[\left\langle S_{x}\right\rangle^{2}+\left\langle S_{y}\right\rangle^{2}\right]^{1 / 2}$ and its explicit expression

$\langle S\rangle=\frac{e E_{e l} m^{*} \tau_{F}}{2 \pi \hbar^{3}} \sqrt{\alpha^{2}+\beta^{2}+2 \alpha \beta \sin (2 \psi)}$,

agrees with that of Ref. [22]. The anisotropy of the spin accumulation is due to the angular dependence of the energy given by Eq. (6): the spin-orbit splitting is different for different directions of the momentum [22]. Fig. 5 shows the dependence of the spin accumulation (in arbitrary units) on the electron concentration of a 2DEG, for equal Rashba and Dresselhaus SOI terms (solid curve, $\alpha=\beta=5 \times 10^{-11} \mathrm{eV} \mathrm{m}$ ), and in the presence of the Rashba SOI term only (dashed curve). The higher values for $\alpha=\beta$ are mainly due to the factor $\left[\alpha^{2}+\beta^{2}+2 \alpha \beta \psi\right]^{1 / 2}$ in Eq. (47).

The transport properties of a Q1DEG have been investigated in the presence of only the Rashba [25] SOI term in the ballistic regime. Here we treat the case when both the Rashba and Dresselhaus SOI terms are present in the non-ballistic regime. We first notice that the non-diagonal conductivity is zero for square and parabolic confinement. As for the expression for the diffusive conductivity, it is easier to evaluate than that of a 2DEG since only the integration over $k_{y}$ needs to be performed. Using Eq. (26) with $\tau$ constant and one subband occupied for square confinement, we obtain, for very low temperatures, the result

$\sigma_{y y}^{d i f}=\frac{2 e^{2} \tau_{F}}{\pi \hbar^{2}}\left[\alpha^{2}+\beta^{2}+4 \lambda\left(E_{F}-E_{1}\right)\right]^{1 / 2}$.

The corresponding density of states reads

$D_{1 \mathrm{D}}(E)=2 /\left(\pi\left[\alpha^{2}+\beta^{2}+4 \lambda E\right]^{1 / 2}\right)$

For parabolic confinement and equal Rashba and Dresselhaus SOI strengths $(\alpha=\beta)$ the conductivity is

where $E_{n}^{0}=(n+1 / 2) \hbar \omega$. This result is also valid for $\alpha-\beta$. The conductivity is shown in Fig. 6 as a function of the electron concentration for $\alpha=\beta=5 \times 10^{-11} \mathrm{eV} \mathrm{m}$. The non-monotonic dependence is due to the presence of discrete levels in the lateral direction produced by the parabolic confinement. Note that the relaxation time $\tau_{F}$ depends on the concentration. Better insight can

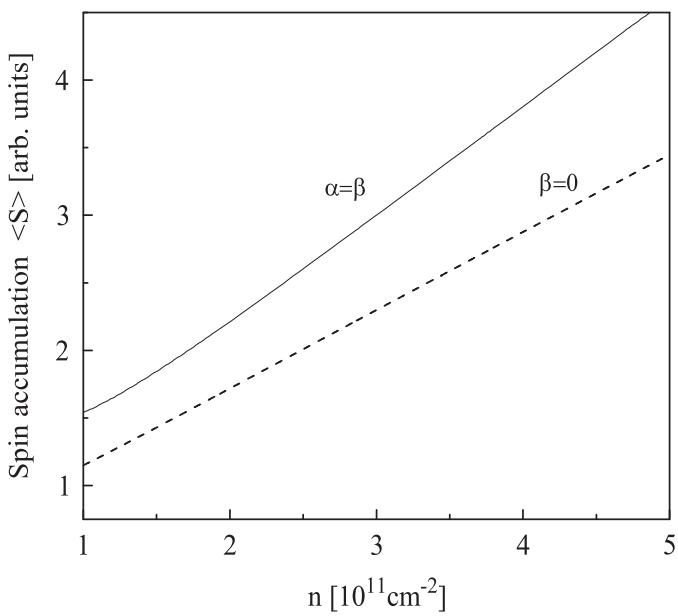

Fig. 5. Spin accumulation in a 2DEG as a function of the electron concentration for $\alpha=\beta=\alpha_{0}=5 \times 10^{-11} \mathrm{eV} \mathrm{m}$ (solid curve) and $\alpha=\alpha_{0}, \beta=0$ (dashed curve) at $T=0 \mathrm{~K}$. 


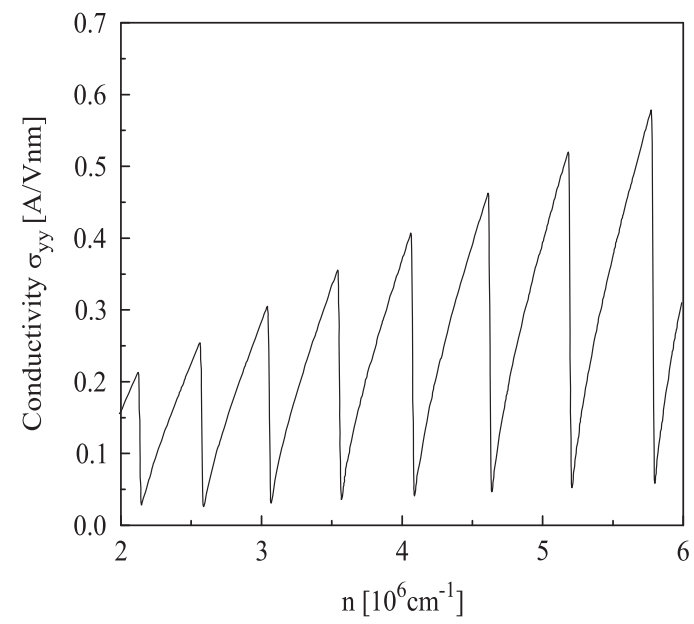

Fig. 6. Diffusive conductivity of a parabolically confined $\mathrm{QW}$ as a function of the electron concentration, $\alpha=\beta=\alpha_{0}=5 \times 10^{-11} \mathrm{eV} \mathrm{m}$.

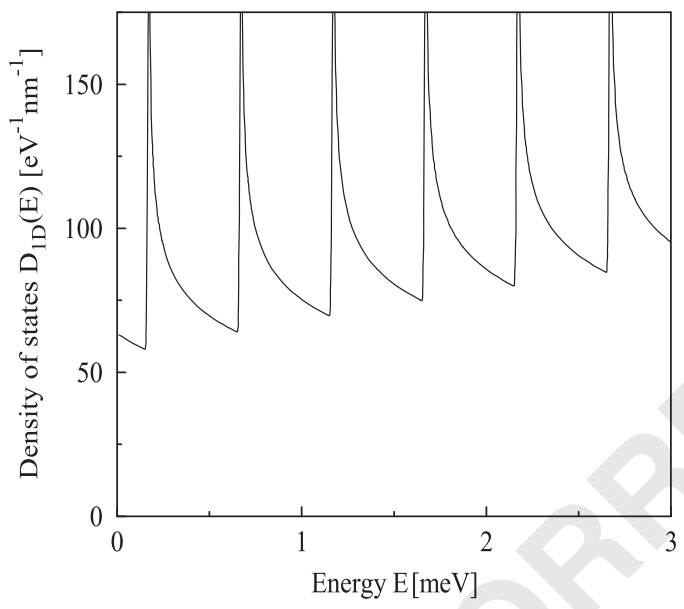

Fig. 7. Density of states of a parabolically confined $\mathrm{QW}$ for $\alpha=\beta=\alpha_{0}=5 \times 10^{-11} \mathrm{eV} \mathrm{m}$.

be acquired by investigating the density of states $D_{1 \mathrm{D}}$ for which the result is

$D_{1 \mathrm{D}}(E)=\frac{\left(2 m^{*}\right)^{1 / 2}}{\pi \hbar} \sum_{n}\left[E-E_{n}^{0}+\alpha^{2} / \lambda\right]^{-1 / 2} ;$

the summation terminates at the last occupied level. $D_{1 \mathrm{D}}(E)$ is shown in Fig. 7 as a function of energy in appropriate units. The usual singularities are visible but shifted by $\alpha^{2} / \lambda$ as a result of the SOI. Notice the correspondence with the maxima of $\sigma_{y y}^{d i f}$.

As in the case of a 2DEG, we will evaluate the spin accumulation using the general expression Eq. (30) for square confinement and only one subband occupied. The result is

$$
\left\langle S_{y}\right\rangle=\frac{e E_{e l} \tau_{F}}{\pi \hbar} \frac{\beta}{\sqrt{\alpha^{2}+\beta^{2}+4 \lambda\left(E_{F}-E_{1}\right)}} .
$$

Note that the net spin in this case depends not only on $\tau_{F}$ but also on $E_{F}$ in contrast to the 2DEG, see Eq. (47). In addition, its value vanishes in the absence of the Dresselhaus SOI. Notice also that while the 2D result Eq. (47) is zero for $\alpha=\beta$ and $\psi=-\pi / 4$, Eq. (52) pertains to quasi-1D systems and for that reason we evaluated only $\left\langle S_{y}\right\rangle$. However, $\sigma_{z^{\prime}}=\left(\sigma_{x}-\sigma_{y}\right) / \sqrt{2}$ is a conserved quantity when $\alpha=\beta$, and the result for $\left\langle S_{z^{\prime}}\right\rangle$ is

$\left\langle S_{z^{\prime}}\right\rangle=\frac{e E_{e l} \tau_{F}}{\pi \hbar} \frac{\alpha-\beta}{\sqrt{\alpha^{2}+\beta^{2}+4 \lambda\left(E_{F}-E_{1}\right)}}$.

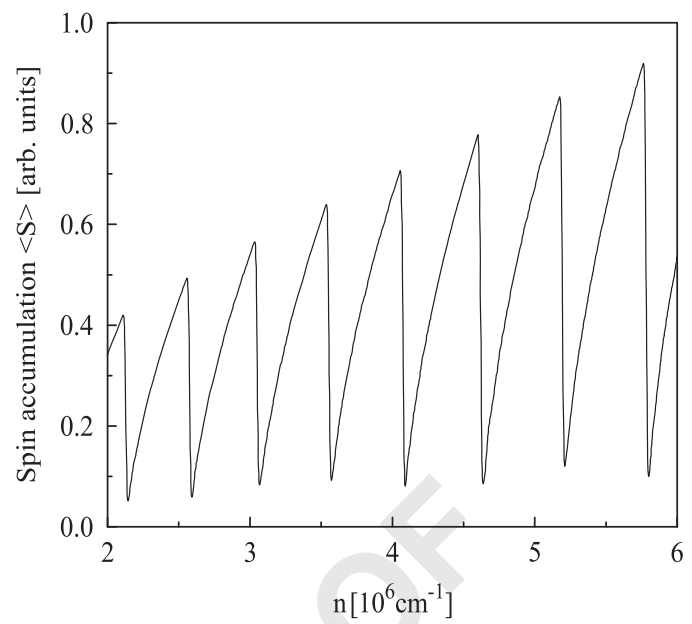

Fig. 8. Spin accumulation in a parabolically confined QW as a function of the electron concentration, at zero temperature, for $\alpha=\beta=\alpha_{0}=5 \times 10^{-11} \mathrm{eV} \mathrm{m}$ and $\hbar \omega=0.5 \mathrm{meV}$.

The quantum wire with parabolic confinement should be treated independently due to the different energy spectrum and eigenvectors. However, analytical results can be found only for equal SOI strengths $\alpha=\beta$. Noticing that the eigenvalues have only quadratic terms in $k_{y}$ leads to

$$
\left\langle S_{y}\right\rangle=\frac{\sqrt{2}}{4} \frac{e E_{e l} \tau_{F}}{\pi \hbar} N_{o c c},
$$

where $N_{\text {occ }}$ is the number of occupied levels. The scattering time $\tau_{F}$ is evaluated assuming a screened Coulomb impurity potential, which for $1 \mathrm{D}$ systems may be modeled [26] as $U(y)=U_{0} e^{-k_{s}|y|}$ $|y|^{1 / 2}$. The spin accumulation also depends on the Fermi level but indirectly through $N_{\text {occ }}$ and the scattering time $\tau_{F}$. In Fig. 8 we show the spin accumulation (in arbitrary units) in a QW with parabolic confinement as a function of the concentration. The steps are due to the non-monotonic dependence of the Fermi level on the concentration, i.e., the occupancies of the discrete levels jump suddenly.

\section{Concluding remarks}

We studied the transport properties of a 2DEG and of QWs, with square or parabolic confinement, in the presence of the Rashba and Dresselhaus terms of the SOI. We derived analytical expressions for the diagonal and non-diagonal conductivities. For a 2DEG the conductivity has an additional term $\propto\left|\alpha^{2}-\beta^{2}\right|$, when compared with previous semiclassical results, that is of quantum mechanical origin. In addition, there is a non-diagonal contribution the conductivity $\propto\left(\alpha^{2}-\beta^{2}\right)^{2}$, cf. Eq. (43), which, however, is very small.

For a QW with square-well confinement the expressions are valid for $\alpha \neq \beta$ but with only the lowest subband occupied while for a parabolically confined QW they are valid only for $\alpha= \pm \beta$ but with no limitation on the number of occupied subbands. We also obtained approximate and numerical results in the latter case for $\alpha \neq \beta$. The diffusive conductivity is given by expressions similar to that for a 2DEG but without the term $\propto\left|\alpha^{2}-\beta^{2}\right|$, cf. Eqs. (49) and (50), but the non-diagonal contribution to the conductivity vanishes.

Moreover, we evaluated the amount of spin accumulation for both a 2DEG and a quasi-1DEG, and discussed its dependence on the SOI strengths and the Fermi energy. Finally, we evaluated the momentum relaxation time, usually taken as constant or evaluated for $\delta$-function potentials, analytically for three type of possible impurity potentials, see Appendix A, and numerically for some results. E.g., those of Fig. 3. 


\section{Acknowledgements}

Our work was supported by the Canadian NSERC Grant no. OGP0121756.

\section{Appendix A. Evaluation of the relaxation time}

The general expression for the relaxation time $\tau$ is

$\frac{1}{\tau_{F}}=\frac{1}{(2 \pi)^{d}} \sum_{\sigma^{\prime}} \int d^{d} \mathbf{k}^{\prime} W_{k k^{\prime} \sigma \sigma^{\prime}}\left(1-\hat{\mathbf{k}} \cdot \hat{\mathbf{k}}^{\prime}\right)$,

where $W_{k k^{\prime} \sigma \sigma^{\prime}}$ is the transition rate between the $|k, \sigma\rangle$ and $\left|k^{\prime}, \sigma^{\prime}\right\rangle$ eigenstates,

$W_{k k^{\prime} \sigma \sigma^{\prime}}=\left(2 \pi n_{i} / \hbar\right)\left|\left\langle k|U| k^{\prime}\right\rangle\right|^{2} \delta_{\sigma \sigma^{\prime}} \delta\left(E_{k}^{\sigma}-E_{k^{\prime}}^{\sigma}\right)$.

In Eq. (A.1) $d$ denotes the dimensionality of the structure: $d=2$ for a 2DEG and $d=1$ for a 1DEG. Further, it is understood that $k$ is the Fermi wave vector as the transport properties are determined by the states near the Fermi energy. The potential is spin-independent; this results in the appearance of $\delta_{\sigma \sigma^{\prime}}$. Eq. (A.1) holds only for elastic or quasi-elastic scattering and under assumption that the relaxation time depends only on the energy of state [20]. The results for several types of impurity potentials are as follows.

$d=2,2 D E G$ : (a) For screened, Coulomb-type impurity potentials, $U(r)=U_{0} e^{-k_{s} r} / r$, with $U_{0}=e^{2} /\left(4 \pi \varepsilon_{0} \varepsilon_{r}\right)$ and $k_{s}$ the screening wave vector, the matrix element $\left\langle k|U| k^{\prime}\right\rangle$ is

$\left\langle k|U| k^{\prime}\right\rangle=\left(2 \pi U_{0}\right)\left(k_{s}^{2}+q^{2}\right)^{-1 / 2}$,

with $q=\left|\mathbf{k}-\mathbf{k}^{\prime}\right|$. The integration over $k^{\prime}$ is carried out using the properties of the $\delta$ function and only the root $k^{\prime}=k$ of the equation $E(k)-E\left(k^{\prime}\right)=0$ contributes to the integral. If the Rashba SOI dominates over the Dresselhaus SOI term, i.e., for $\alpha \gg \beta$, the integration over $\theta$ in Eq. (A.1) can be carried out analytically. The result is

$\frac{1}{\tau_{F}}=\frac{2 \pi^{2} n_{i} U_{0}^{2}}{\hbar}\left(I_{C+}+I_{C-}\right)$,

where

$I_{C+}=\frac{k_{+}}{2 \lambda k_{+}+\alpha} \int_{0}^{2 \pi} \frac{(1-\cos \theta) d \theta}{k_{\mathrm{s}}^{2}+q^{2}}$

and $q^{2}=2 k_{+}^{2}(1-\cos \theta)$. Note that $n_{i}$ is the concentration of impurities per unit area. By contour integration the integral over $\theta$ gives $\left(\pi / k_{+}^{2}\right)\left[1-k_{s} /\left(k_{s}^{2}+4 k_{+}^{2}\right)^{1 / 2}\right]$ and one obtains

$I_{C+}=\left[1-k_{S} /\left(4 k_{+}^{2}+k_{s}^{2}\right)^{1 / 2}\right] /\left[k_{+}\left(2 \lambda k_{+}+\alpha\right)\right]$.

$I_{C_{-}}$is determined by the same formula with $2 \lambda k_{+}+\alpha$ replaced by $\left|2 \lambda k_{-}-\alpha\right|$ in Eq. (A.6).

(b) For short-range impurity potentials of the form $U(\mathbf{r})=V_{0} \delta\left(\mathbf{r}-\mathbf{r}_{\mathbf{i}}\right)$, where $\mathbf{r}_{i}$ is the position of the impurity, $\tau_{F}$ is easier to evaluate and the matrix element $\left|\left\langle k|U| k^{\prime}\right\rangle\right|^{2}$ is simply equal to $V_{0}^{2}$. This leads to

$\frac{1}{\tau_{F}}=\frac{2 m^{*} n_{i} V_{0}^{2}}{\hbar^{3}}$,

where $V_{0}$ has the units of energy times length squared.

(c) For Gaussian-type impurity potentials, of the form $U(r)=$ $U_{0} e^{-r^{2} / 2 \sigma^{2}}$, one readily obtains

$\left\langle k|U| k^{\prime}\right\rangle=2 \pi U_{0} \sigma^{2} e^{-\left|\mathbf{k}-\mathbf{k}^{\prime}\right|^{2} \sigma^{2} / 2}$

Then Eq. (A.1) assumes the form

$\frac{1}{\tau_{F}}=\frac{2 \pi n_{i} U_{0}^{2} \sigma^{4}}{\hbar} \int_{0}^{2 \pi} d \theta \int_{0}^{\infty} d k^{\prime}(1-\cos \theta) e^{-\left|\mathbf{k}-\mathbf{k}^{\prime}\right|^{2} \sigma^{2}}$ $\times \delta\left(\lambda\left(k^{2}-k^{\prime 2}\right) \pm \alpha\left(k-k^{\prime}\right)\right)$.
Only the root $k=k^{\prime}$ contributes to the integral over $k^{\prime}$. Then, after integration over $\theta$, Eq. (A.9) takes the form

$\frac{1}{\tau_{F}}=\frac{2 \pi n_{i} U_{0}^{2} \sigma^{4}}{\hbar}\left(I_{G+}+I_{G-}\right)$,

where the "+" branch gives

$I_{G+}=\frac{2 \pi k_{+}}{2 \lambda k_{+}+\alpha}\left[I_{0}\left(2 \sigma^{2} k_{+}^{2}\right)-I_{1}\left(2 \sigma^{2} k_{+}^{2}\right)\right]$,

with $I_{0}$ and $I_{1}$ being the zeroth- and first-order modified Bessel functions, respectively. The "-" branch gives Eq. (A.11) with $2 \lambda k_{+}+\alpha$ replaced by $\left|2 \lambda k_{-}-\alpha\right|$.

$d=1,1 D E G$ : (d) For a QW with parabolic confinement, $\alpha=\beta$, and screened Coulomb impurities we consider the model potential [26]

$U(r)=U_{0} \exp \left(-k_{s}|y|\right) /|y|^{1 / 2}$,

with $U_{0}=2 \pi \sqrt{c} e^{2} / \varepsilon_{0} \varepsilon_{r}$ and $c$ a material-dependent parameter [26]. Since $1-\hat{\mathbf{k}} \hat{\mathbf{k}}^{\prime}$ is nonzero only if $k_{y}^{\prime}=-k_{y}$. Then Eq. (A.1) takes the form $\frac{1}{\tau_{F}}=\frac{1}{2 \pi} \sum_{n, \sigma} \int_{-\infty}^{\infty} 2 W_{k k^{\prime}} d k^{\prime}$

and

$\left\langle-k_{y n}^{\prime}|U| k_{y n}^{\prime}\right\rangle=\sqrt{\pi} U_{0}\left(\left[k_{s}-2 i k_{y n}^{\prime}\right]^{-1 / 2}+\left[k_{s}+2 i k_{y n}^{\prime}\right]^{-1 / 2}\right)$.

The final result is

$\frac{1}{\tau_{F}}=\frac{8 \pi m^{*} n_{i} U_{0}^{2}}{\hbar^{3}} \sum_{n} \frac{k_{s}+\sqrt{k_{s}^{2}+4 k_{y n}^{2}}}{k_{y n}\left(k_{s}^{2}+4 k_{y n}^{2}\right)}$

Once again, $k_{y n}$ in the last equation denotes the Fermi wave vector for the $n$th subband. The wave vector of the last subband is small and contributes the most to the value of $\tau_{F}$, so that the sum in Eq. (A.15) can, in practice, be approximated by a single term $n=N_{\text {occ }}$.

(e) For a QW with parabolic confinement, $\alpha=\beta$, and a shortrange impurity potential $U(r)$ given by $U(r)=V_{0} \delta\left(y-y_{i}\right)$ we proceed as in the case $d=2$. The matrix element of this potential is now easier to evaluate, the result is $\left|\left\langle-k_{y}^{\prime}|U(y)| k_{y}^{\prime}\right\rangle\right|^{2}=V_{0}^{2}$. This leads to a simple relation for the relaxation time at the Fermi energy

$\frac{1}{\tau_{F}}=\frac{4 m^{*} n_{i} V_{0}^{2}}{\hbar^{3}} \sum_{n} \frac{1}{k_{y n}}$

(f) For QW with parabolic confinement, $\alpha=\beta$, and a Gaussian impurity potential of the form $U(r)=U_{0} e^{-\left(y-y_{i}\right)^{2} / 2 \sigma^{2}}$, we easily obtain $\left|\left\langle-k_{y}^{\prime}|U(y)| k_{y}^{\prime}\right\rangle\right|^{2}=2 \pi \sigma^{2} U_{0}^{2} e^{-2 k_{y}^{2} \sigma^{2}}$. This yields

$\frac{1}{\tau_{F}}=\frac{8 \pi m^{*} n_{i} U_{0}^{2} \sigma^{2}}{\hbar^{3}} \sum_{n} \frac{e^{-2 k_{y n}^{2} \sigma^{2}}}{k_{y n}}$

\section{References}

[1] M.N. Baibich, J.M. Broto, A. Fert, F.N. Van Dau, F. Petroff, P. Etienne, G. Creuzet A. Friederich, J. Chazelas, Phys. Rev. Lett. 61 (1988) 2472

[2] I. Žutić, J. Fabian, S. Das Sarma, Rev. Mod. Phys. 76 (2004) 323.

[3] Y.A. Bychkov, E.I. Rashba, J. Phys. C 17 (1984) 6039.

[4] R. Winkler, Spin-Orbit Coupling Effects in Two-dimensional Electron and Hole Systems, Springer Tracts in Modern Physics, vol. 191, Springer, New York, 2003.

[5] G. Dresselhaus, Phys. Rev. 100 (1955) 580.

[6] J. Nitta, T. Akazaki, H. Takayanagi, T. Enoki, Phys. Rev. Lett. 78 (1997) 1335; C.-M. Hu, J. Nitta, T. Akazaki, H. Takayanagi, J. Osaka, P. Pfeffer, W. Zawadzki, Phys. Rev. B 60 (1999) 7736.

[7] G. Engels, J. Lange, T. Schäpers, H. Lüth, Phys. Rev. B 55 (1997) R1958.

[8] D. Grundler, Phys. Rev. Lett. 84 (2000) 6074.

[9] W. Yang, K. Chang, Phys. Rev. B 73 (2006) 045303; X.F. Wang, P. Vasilopoulos, Phys. Rev. B 67 (2003) 085313.

[10] J. Inoue, G.W. Bauer, L.W. Molenkamp, Phys. Rev. B 67 (2003) 033104

[11] W. Knap, et al., Phys. Rev. B 53 (1996) 3912.

[12] J. Schliemann, J.C. Egues, D. Loss, Phys. Rev. Lett. 90 (2003) 146801 
1 [13] J.D. Koralek, C.P. Weber, J. Orenstein, B.A. Bernevig, S.-C. Zhang, S. Mack, D.D. Awschalom, Nature 458 (2009) 610.

[14] B.A. Bernevig, J. Orenstein, S.-C. Zhang, Phys. Rev. Lett. 97 (2006) 236601; X. Cartoixà, L.-W. Wang, D.Z.-Y. Ting, Y.-C. Chang, Phys. Rev. B 73 (2006) 205341;

M.-H. Liu, K.-W. Chen, S.-H. Chen, C.-R. Chang, Phys. Rev. B 74 (2006) 235322.

[15] A.A. Reynoso, G. Usaj, C.A. Balseiro, Phys. Rev. B 78 (2008) 115312.

[16] P.M. Krstajić, E. Rezasoltani, P. Vasilopoulos, Phys. Rev. B 81 (2010) 155325

[17] A.V. Moroz, C.H.W. Barnes, Phys. Rev. B 60 (1999) 14272

[18] S. Debald, B. Kramer, Phys. Rev. B 71 (2005) 115322.
[19] S. Zhang, R. Liang, E. Zhang, L. Zhang, Y. Liu, Phys. Rev. B 73 (2006) 155316.

[20] M. Charbonneau, K.M. Van Vliet, P. Vasilopoulos, J. Math. Phys. 23 (1982) 318.

[21] F. Reif, Fundamentals of Statistical and Thermal Physics, McGraw-Hill, New York, 1965

[22] M. Trushin, J. Schliemann, Phys. Rev, B 75 (2007) 155323.

[23] J. Schliemann, D. Loss, Phys. Rev. B 68 (2003) 165311.

[24] S.A. Tarasenko, N.S. Averkiev, JETP Lett. 75 (2002) 669.

[25] F. Mireles, G. Kirczenow, Phys. Rev. B 64 (2001) 024426.

[26] P. Vasilopoulos, F.M. Peeters, Phys. Rev. B 40 (1989) 10079. 